\title{
Não monotonicidade do parâmetro crítico no modelo dos sapos
}

\author{
Alexandre Ribeiro Leichsenring \\ DISSERTAÇÃO APRESENTADA \\ AO INSTITUTO DE MATEMÁTICA E ESTATÍSTICA \\ DA UNIVERSIDADE DE SÃO PAULO \\ PARA OBTENÇÃO \\ DO GRAU DE MESTRE EM ESTATÍSTICA \\ Área de Concentração: Probabilidade \\ Orientador: Prof. Dr. Fábio Prates Machado
}

São Paulo, 18 de Fevereiro de 2003.

O autor agradece ao CNPq pelo apoio financeiro (Processo N. 131546/2001-5). 


\section{Resumo}

Estudamos um modelo de passeios aleatórios simples em grafos, conhecido como modelo dos sapos. Esse modelo pode ser descrito de maneira geral da seguinte forma: existem partículas ativas e partículas desativadas num grafo $\mathcal{G}$. Cada partícula ativa desempenha um passeio aleatório simples a tempo discreto e a cada momento ela pode morrer com probabilidade 1-p. Quando uma partícula ativa entra em contato com uma partícula desativada, esta é ativada e também passa a realizar, de maneira independente, um passeio aleatório pelo grafo. Apresentamos limites superior e inferior para o parâmetro crítico de sobrevivência do modelo dos sapos na árvore, e demonstramos que este parâmetro crítico não é uma função monótona do grafo em que está definido. 


\begin{abstract}
We study a system of simple random walks on graphs, known as frog model. This model can be described generally speaking as follows: there are active and sleeping particles living on some graph $\mathcal{G}$. Each particle performs a simple random walk with discrete time and at each moment it may disappear with probability $1-p$. When an active particle hits a sleeping particle, the latter becomes active and starts to perform, independently, a simple random walk on the graph. We present lower and upper bounds for the surviving critic parameter on the tree, and we show that this parameter is not a monotonic function of the graph it is defined on.
\end{abstract}




\section{Índice}

1 Introdução 4

2 Processo de Ramificação $\quad 6$

$\begin{array}{llr}3 & \text { Acoplamento } & 10\end{array}$

4 O modelo dos Sapos $\quad 14$

4.1 Transição de fase . . . . . . . . . . . . 16

4.2 Não Monotonicidade ................... 21

$\begin{array}{lr}\text { Referências Bibliográficas } & 28\end{array}$ 


\section{Introdução}

Os sistemas de partículas interagentes são um ramo da teoria da probabilidade que reune modelos suficientemente flexíveis para modelar sistemas reais e imaginários. Nestes modelos, partículas evoluem no espaço e no tempo, seguindo regras simples de interação local, levando em conta essencialmente o que ocorre apenas dentro de uma vizinhança finita tomada em relação à posição no espaço em que se encontram essas partículas (os livros de Liggett [8] e [9] e o de Durrett [6] são importantes referências teóricas). Parte do atrativo teórico destes modelos está no fato de combinarem uma dinâmica local bastante simples com um comportamento global espacial e temporal bastante rico e cheio de surpresas - um campo fértil para a aplicação e desenvolvimento da teoria da probabilidade. Do ponto de vista das aplicações, o que chama a atenção é uma rica conexão com outras áreas da ciência. Inicialmente desenvolveu-se uma interface bastante ampla com a Física, mas à medida que seu estudo foi evoluindo, descobriu-se generosas possibilidades de aplicações na Biologia, Ciências Sociais e Economia para citar algumas áreas. Entre várias possibilidades, os sistemas de partículas interagentes que são tipos de processos estocásticos - podem ser usados para modelar magnetismo, competição entre organismos por espaço físico, crescimento de tumores, espalhamento de infecções ou mesmo sistemas econômicos.

Neste trabalho estudamos questões assintóticas qualitativas de um sistema de passeios aleatórios em grafos conhecido como modelo dos sapos. Este modelo tem recebido boa dose de atenção recentemente em função de 
sua simplicidade e flexibilidade, aliados à possibilidade de um amplo leque de tratamento teórico que pode receber. Para citar alguns exemplos mencionamos teorema da forma, transição de fase, limite hidrodinâmico e densidade assintótica.

A dinâmica do sistema estudado neste trabalho pode ser descrita de modo geral da seguinte maneira. Em cada sítio do grafo $\mathcal{G}$ encontra-se um número $\eta$ (aleatório) de partículas. Escolhemos um sítio e o denominamos origem. Inicialmente todas as partículas encontram-se dormentes, exceto por aquelas situadas à origem. Em cada passo, cada partícula ativa morre com probabilidade $1-p$ num processo que evolui em tempo discreto. Uma vez que uma partícula sobrevive, ela salta para um dos sítios vizinhos, escolhidos com probabilidade uniforme, executando um passeio aleatório simples nos sítios de $\mathcal{G}$. Não há interação entre as partículas ativas, o que quer dizer que elas realizam passeios aleatórios independentes. A partir do momento em que uma partículas dormente é ativada ela passa a desempenhar pelo grafo um passeio aleatório independente, ativando todas as partículas desativadas que encontra pelo caminho, até o momento em que ela morre.

Os resultados apresentados aqui são motivados por um trabalho que está sendo escrito por L. Fontes, F. Machado e A. Sarkar.

Nas duas próximas seções introduzimos duas ferramentas centrais utilizadas nesse estudo, a saber, os processos de ramificação e a técnica de acoplamento. Na última seção apresentamos limites inferior e superior para o valor do parâmetro crítico de sobrevivência do modelo dos sapos no grafo conhecido como árvore, e demonstramos que o parâmetro crítico do modelo 
dos sapos, ao contrário do que ocorre no modelo de percolação, não é uma função monotônica do grafo. Isto é, se $\mathcal{G}_{1}$ e $\mathcal{G}_{2}$ são dois grafos tais que $\mathcal{G}_{1} \subset \mathcal{G}_{2}$ não decorre daí que $p_{c}\left(\mathcal{G}_{1}\right) \geq p_{c}\left(\mathcal{G}_{2}\right)$, o que poderia ser esperado dado que estamos falando de um modelo de percolação.

\section{Processo de Ramificação}

Os processos de ramificaçao são bastante úteis no estudo dos sistemas de partículas. Os motivos disto são a naturalidade e simplicidade com que certos modelos de sistemas de partículas podem ser comparados com alguns processos de ramificação e o fato de que estes são bem compreendidos. Comparações do modelo dos sapos com processos de ramificação serão utilizadas mais de uma vez nesta dissertação através da técnica de acoplamento, descrita na próxima seção.

Os processos de ramificação são estudados há pelo menos 30 anos. O famoso livro de Athreya e Ney [4] é uma ótima referência para a teoria desses processos. Com o objetivo de tornar este texto auto contido apresentamos nas linhas que seguem um pequeno sumário das propriedades básicas dos processos de ramificação, embora todos os fatos apresentados e utilizados aqui sejam bem divulgados nos livros sobre teoria das probabilidades.

Suponha, então, que $\{f(n), n \geq 0\}$ seja uma densidade de probabilidade sobre os inteiros não negativos. Construímos uma cadeia de Markov discreta $X_{n}$ em $\{0,1, \ldots\}$ fazendo a distribuição condicional de $X_{n+1}$ dado $X_{n}=k$ igual à distribuição da soma de $k$ variáveis aleatórias independentes com 
densidade $f$. Então $X_{n}$ pode ser interpretada como o número de indivíduos na n-ésima geração de uma população em que cada membro se reproduz em tempos discretos gerando um número aleatório de descendentes, escolhido com densidade $f$.

Denotamos o número inicial de indivídos por $X_{0}$. Vamos demonstrar que a população se extingue com probabilidade 1 se e somente se a esperança do número de filhos de cada indivíduo é menor ou igual a 1 - talvez este seja o resultado mais conhecido da teoria dos processos de ramificação. Se $f(0)=1$ então o processo morre no primeiro passo. Por outro lado, se $f(0)=0$ temos que $X_{n}$ é sempre não decrescente em $n$. Para evitar esse tipo de trivialidade vamos supor $0<f(0)<1$ e $f(0)+f(1)<1$.

Vamos também supor $X_{0}=1$ e seja $\mu$ o número médio de descendentes por indivíduo.

Vamos chamar de $\pi_{0}$ a probabilidade de, começando com 1 indivíduo, a população se extinguir. Observe que

$$
\begin{aligned}
\pi_{0} & =\mathbf{P}\{\text { população se extinguir }\} \\
& =\sum_{j=0}^{\infty} \mathbf{P}\left\{\text { população se extinguir } \mid X_{1}=j\right\} f(j) .
\end{aligned}
$$

Agora, dado que $X_{1}=j$, para que a população se extinga é preciso que cada uma das $j$ sequências evoluindo independentemente se extingam. Como a probabilidade de uma família particular se extinguir é $\pi_{0}$, temos 


$$
\pi_{0}=\sum_{j=0}^{\infty} \pi_{0}^{j} f(j)
$$

Vamos definir a função geradora de probabilidade de $f$ :

$$
\phi(s)=\sum_{j=0}^{\infty} s^{j} f(j)
$$

Assim, temos

$$
\phi^{\prime}(s)=\frac{d}{d s} \phi(s)=\sum_{j=1}^{\infty} j f(j) s^{j-1} .
$$

Como $\phi^{\prime}(s)>0$ para $0<s<1$, $\phi$ é estritamente crescente. Além disso, como $\phi^{\prime \prime}(s)$ satisfaz

$$
\phi^{\prime \prime}(s)=\frac{d^{2}}{d s^{2}} \phi(s)=\sum_{j=2}^{\infty} j(j-1) f(j) s^{j-2}>0 \text { para } 0<s<1,
$$

a função $\phi$ é estritamente convexa em $(0,1)$. Como $\phi(0)>0$ e $\phi(1)=$ $\sum_{j=0}^{\infty} f(j)=1$, o gráfico de $\phi$ se assemelha ao da Figura 1 (curva a ou b).

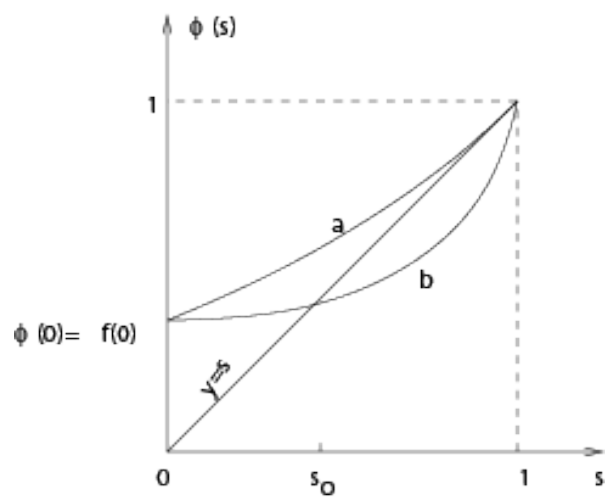

Figura 1: Função geradora de probabilidade 
O máximo de $\phi^{\prime}(s)$ é $\phi^{\prime}(1)=\sum_{j=1}^{\infty} j f(j)=\mu$. Assim, no caso de $\mu>1$, o gráfico de $y=\phi(s)$ deve estar abaixo do de $y=s$ próximo de $s=1 \mathrm{e}$, como $\phi(0)=f(0)>0$, deve cruzar a linha $y=s$ num ponto $s_{0}, 0<s_{0}<1$. Como a inclinação da curva $y=\phi(s)$ cresce constantemente quando $s$ cresce em $(0,1), s_{0}$ é a única solução da equação $s=\phi(s)$ que é menor do que 1.

No caso em que $\mu \leq 1, y=\phi(s)$ deve ficar estritamente acima da linha $y=s$ exceto em $s=1$, pois se $\phi(s)$ cruzar a linha $y=s$ num ponto $s_{0}<1$ ela deveria passar por baixo da linha na vizinhança imediata à direita de $s_{0}$, já que nesse ponto a inclinação de $\phi(s)$ é menor do que a da reta, e então, para que atingisse a altura $\phi(1)=1$ sua inclinação deveria ultrapassar 1 em algum ponto em $\left(s_{0}, 1\right]$; isso seria uma contradição pois $\phi^{\prime}(s) \leq \phi^{\prime}(1)=\mu$ para todo $s$ em $[0,1]$. Logo, a única solução da equação $s=\phi(s)$ é $s=1$.

De posse disso, podemos provar o seguinte teorema:

Teorema 2.1 O processo morre com probabilidade 1 se e somente se $\mu \leq 1$.

Demostração Observe que $\pi_{0}=\phi\left(\pi_{0}\right)$. Se $\mu \leq 1$ então $\pi_{0}=1$ e a extinção é certa.

Suponha agora $\mu>1$. Então $\pi_{0}$ é ou $s_{0}(<1)$ ou 1 . Seja $\pi>0$ tal que $\pi=\phi(\pi)$. Basta mostrar que $\pi \geq \pi_{0}$. Primeiro mostraremos por indução que $\pi \geq \mathbf{P}\left\{X_{n}=0\right\}$ para todo $\mathrm{n}$.

$$
\pi=\sum_{j=0}^{\infty} \pi^{j} f(j) \geq \pi^{0} f(0)=f(0)=\mathbf{P}\left\{X_{1}=0\right\}
$$

Agora assuma que $\pi \geq \mathbf{P}\left\{X_{n}=0\right\}$. Então 


$$
\begin{aligned}
\mathbf{P}\left\{X_{n+1}=0\right\} & =\sum_{j=0}^{\infty} \mathbf{P}\left\{X_{n+1}=0 \mid X_{1}=j\right\} f(j) \\
& =\sum_{j=0}^{\infty}\left(\mathbf{P}\left\{X_{n}=0\right\}\right)^{j} f(j) \\
& \leq \sum_{j=0}^{\infty} \pi^{j} f(j) \quad \text { (pela hipótese de indução) } \\
& =\pi
\end{aligned}
$$

Daí,

$$
\pi \geq \mathbf{P}\left\{X_{n}=0\right\} \quad \text { para todo } n
$$

e fazendo $n \longrightarrow \infty$, temos

$$
\pi \geq \lim _{n} \mathbf{P}\left\{X_{n}=0\right\}=\mathbf{P}\{\text { população se extinguir }\}=\pi_{0} .
$$

\section{Acoplamento}

Acoplamento é uma técnica bastante importante e aplicável com grande generalidade nos sistemas de partículas interagentes. Apesar da técnica preceder o tema, foi graças ao seu poder e flexibilidade na teoria de sistemas de partículas interagentes que ela ganhou mais reconhecimento durante a década de 1970. O livro de Thorisson [13] é uma excelente referência. Devido ao importante papel que essa técnica desenvolve nesta dissertação, apresentamos agora um breve sumário de seu conceito e algumas aplicações. 
Acoplamento significa a construção conjunta de duas ou mais variáveis (ou processos) aleatórias(os) num espaço de probabilidade comum, normalmente com a intenção de deduzir propriedades das variáveis individuais ou da relação entre elas.

Um exemplo de uso bastante eficiente de acoplamento é o seguinte. Suponha que se deseja provar a afirmação bastante plausível de que para toda variável aleatória $X$ e funções $f$ e $g$ não decrescentes na reta real, as variáveis aleatórias $f(X)$ e $g(X)$ são positivamente correlacionadas. A prova é bastante simples se contarmos com o auxílio de uma variável aletória $X^{\prime}$, cópia independente de $X$. Por causa da monotonicidade de $f$ e $g$ devemos ter o produto $\left[f(X)-f\left(X^{\prime}\right)\right]\left[g(X)-g\left(X^{\prime}\right)\right] \geq 0$. Assim,

$$
\begin{aligned}
0 & \leq \mathbf{E}\left[f(X)-f\left(X^{\prime}\right)\right]\left[g(X)-g\left(X^{\prime}\right)\right] \\
& =\mathbf{E}[f(X) g(X)]-\mathbf{E}\left[f(X) g\left(X^{\prime}\right)\right]-\mathbf{E}\left[f\left(X^{\prime}\right) g(X)\right]+\mathbf{E}\left[f\left(X^{\prime}\right) g\left(X^{\prime}\right)\right] \\
& =2\{\mathbf{E}[f(X) g(X)]-\mathbf{E}[f(X)] \mathbf{E}[g(X)]\} \\
& =2 \operatorname{Cov}\{f(X), g(X)\},
\end{aligned}
$$

Nessa desigualdade é importante o fato de $R^{1}$ ser totalmente ordenado, de forma que, com probabilidade 1 , ou $X \geq X^{\prime}$ ou $X \leq X^{\prime}$, e daí $\left[f(X)-f\left(X^{\prime}\right)\right]$ e $\left[g(X)-g\left(X^{\prime}\right)\right]$ têm o mesmo sinal. A desigualdade de FKG representa uma versão do resultado acima para o caso em que o espaço onde $f$ e $g$ estão definidas é apenas parcialmente ordenado.

O exemplo a seguir aplica a técnica de acoplamento para demonstrar o teorema de convergência básico para cadeias de Markov com espaço de 
estados finito. Seja $p(x, y)$ a probabilidade de transição de $x$ para $y$ para uma cadeia de Markov a tempo discreto com espaço de estados definido num conjunto finito $\mathrm{S}$ e seja $p^{k}(x, y)$ a correspondente probabilidade de transição em $k$ passos definidada por:

$$
\begin{aligned}
p^{(1)}(x, y) & =p(x, y) \\
p^{(k+1)}(x, y) & =\sum_{z \in S} p^{(k)}(x, z) p(z, y) .
\end{aligned}
$$

Teorema 3.1 Suponha que para algum $m \geq 1$,

$$
\min _{x, y \in S} p^{(m)}(x, y)=\varepsilon>0
$$

Então

$$
\pi(y)=\lim _{k \rightarrow \infty} p^{(k)}(x, y)
$$

existe para $x, y \in S$, é independente de $x$, e satisfaz

$$
\sum_{x} \pi(x) p(x, y)=\pi(y)
$$

Demostração Seja $\left(X_{n}, Y_{n}\right)$ uma cadeia de Markov em $S \times S$ que evolui da seguinte maneira:

(a) $X_{n}$ e $Y_{n}$ se movem independentemente com probabilidades de transição $p(\cdot, \cdot)$ até o primeiro momento que $X_{n}=Y_{n}$, e

(b) $X_{n}$ e $Y_{n}$ se movem juntos com mesma probabilidade de transição após esse tempo. 
Podemos descrever o processo $\left(X_{n}, Y_{n}\right)$ de maneira equivalente apresentando suas probabilidades de transição:

$$
\mathbf{P}^{(x, y)}\left[X_{1}=u, Y_{1}=v\right]= \begin{cases}p(x, u) p(y, v) & \text { se } x \neq y, \\ p(x, u) & \text { se } x=y \text { e } u=v, \\ 0 & \text { se } x=y \text { e } u \neq v .\end{cases}
$$

Esta cadeia de Markov acoplada tem duas impostantes propriedades:

(a) $X_{n}$ e $Y_{n}$ são separadamente Markovianos com probabilidades de transição $p(\cdot, \cdot), \mathrm{e}$

(b) $\lim _{n \rightarrow \infty} \mathbf{P}^{(x, y)}\left[X_{n}=Y_{n}\right]=1$ para todo $(x, y) \in S \times S$.

Para a primeira propriedade basta ver que as probabilidades marginais de transição de $X_{n}$ e $Y_{n}$ são ambas dadas por $p(\cdot, \cdot)$. Para checar a segunda, seja $\tau$ o tempo em que as duas coordenadas do processo se igualam pela primeira vez. Então

$$
\begin{aligned}
\mathbf{P}^{(x, y)}(\tau \leq m) & \geq \sum_{z \in S} p^{(m)}(x, z) p^{(m)}(y, z) \\
& \geq \varepsilon \sum_{z \in S} p^{(m)}(y, z)=\varepsilon
\end{aligned}
$$

para todo $(x, y) \in S \times S$, portanto pela propriedade Markoviana,

$$
\mathbf{P}^{(x, y)}(\tau \leq k m) \geq 1-(1-\varepsilon)^{k},
$$

e assim 


$$
\mathbf{P}^{(x, y)}\left[X_{n} \neq Y_{n}\right] \leq \mathbf{P}^{(x, y)}(\tau>n) \leq(1-\varepsilon)^{[n / m]},
$$

que tende a zero quando $n \rightarrow \infty$ exponencialmente rápido. Pela propriedade (a) do acoplamento,

$$
\begin{aligned}
\left|p^{(k)}(u, y)-p^{(k)}(v, y)\right| & \left.=\mid \mathbf{P}^{(u, v)}\left[X_{k}=y\right]-\mathbf{P}^{(} u, v\right)\left[Y_{k}=y\right] \mid \\
& =\left|\mathbf{P}^{(u, v)}\left[X_{k}=y, Y_{k} \neq y\right]-\mathbf{P}^{(u, v)}\left[X_{k} \neq y, Y_{k}=y\right]\right| \\
& \leq \mathbf{P}^{(u, v)}\left[X_{k} \neq Y_{k}\right],
\end{aligned}
$$

que tende a zero pela propriedade (b). Como $\max _{x} p^{(k)}(x, y)$ decresce e $\min _{x} p^{(k)}(x, y)$ cresce em $k$ para cada $y$, a prova de 3.2 agora é fácil. A afirmação 3.3 segue tomando o limite em 3.1 quando $k \rightarrow \infty$.

Na seção seguinte tratamos dos resultados da dissertação. A estratégia para obtenção desses resultados é acoplar ao modelo dos sapos processos de ramificação cujo número de descendentes em cada geração, segundo a conveniência, dominam ou são dominados pelo número de partículas ativas em cada instante no modelo dos sapos. A partir desta comparação, extraímos informações a respeito do modelo dos sapos usando os fatos apresentados sobre o comportamento assintótico dos processos de ramificação.

\section{O modelo dos Sapos}

Apresentadas as ferramentas partimos para o estudo do modelo dos sapos, descrito anteriormente. Esse modelo com $p=1$ (i.e., sem mortes) é uma 
versão em tempo discreto do modelo para difusão de informação proposto por R. Durret e K. Ravishankar em 1996. Neste modelo a idéia é que cada partícula ativa possui uma certa informação que é transmitida às partículas dormentes no momento em que a primeira salta sobre estas últimas. O primeiro resultado publicado sobre esse modelo é devido a Telcs e Wormald, no qual foi referido como egg model. Eles provaram que, partindo da configuração inicial com 1 partícula por sítio, a origem é visitada infinitas vezes quase certamente. Popov [12] provou o mesmo para dimensão $d \geq 3$ para a configuração inicial construída da seguinte maneira: uma partícula é colocada em cada $x \neq 0$ com probabilidade $\alpha /\|x\|^{2}$, onde $\alpha$ é uma constante positiva grande. Em Alves et al. [2] foi provado para o modelo dos sapos com configuração inicial de 1 partícula por sítio, que o conjunto das posições originais de todas as partículas ativas, reescalado pelo tempo transcorrido, converge para um conjunto não aleatório compacto convexo não vazio, resultado conhecido como teorema da forma. Em Alves et al. [1] foi demonstrado o teorema da forma para o caso de configuração inicial aleatória, e em Alves et al. [3] foram apresentados para $\mathbb{Z}^{d}$ e para árvores regulares resultados sobre transição de fase e valores assintóticos para parâmetros críticos. O objetivo do presente trabalho é estudar no modelo com morte alguns aspectos da dinâmica assintótica desse modelo de sistema de partículas, com configuração inicial de uma partícula por sítio, com relação ao parâmetro de sobrevivência $p$ e ao grafo onde os passeios aleatórios ocorrem.

Primeiramente apresentamos um estudo sobre a evolução do modelo em grafos conhecidos como árvores com o objetivo de apresentar dois grafos $\mathcal{G}_{1}$ 
e $\mathcal{G}_{2}$, com $\mathcal{G}_{1} \subset \mathcal{G}_{2}$ e tal que $p_{c}\left(\mathcal{G}_{2}\right) \leq p_{c}\left(\mathcal{G}_{1}\right)$. Apresentamos agora a definição formal de árvore.

A árvore $\mathbf{T}_{d}=(\mathcal{V}, \mathcal{E})$ é formada por um conjunto de elos $\mathcal{E}=\mathcal{E}\left(\mathbf{T}_{d}\right)$ e de vértices (ou sítios) $\mathcal{V}=\mathcal{V}\left(\mathbf{T}_{d}\right)$ que se relacionam da seguinte forma: de cada vértice de $\mathcal{V}$ partem $d+1$ elos. Escolhemos um dos vértices e denominamo-no origem. Dois sítios são ditos vizinhos se eles pertencem a um elo comum. Assim, $\mathbf{T}_{d}$ é um grafo conexo infinito sem ciclos em que cada vértice de $\mathcal{V}$ $\left(\right.$ ou $\mathcal{V}\left(\mathbf{T}_{d}\right)$ ) possui $d+1$ vizinhos.

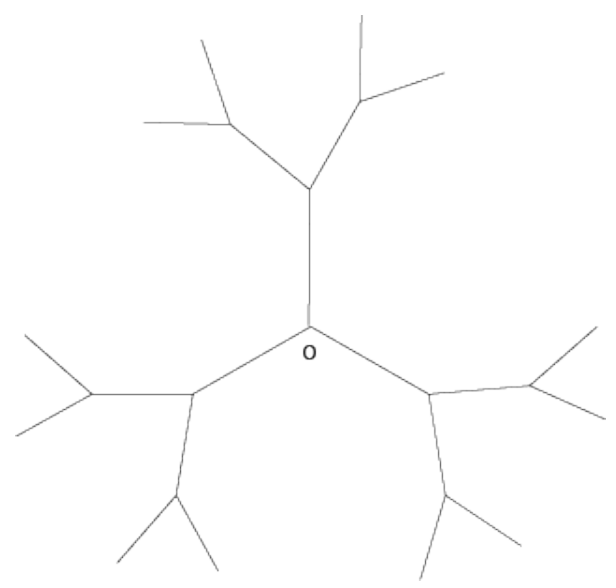

Figura 2: Árvore $\mathbf{T}_{2}$

\subsection{Transição de fase}

A principal quantidade de interesse nesse trabalho é a probabilidade do processo sobreviver, termo que definimos formalmente abaixo.

Definição 4.1 Dizemos que uma realização particular do modelo dos sapos 
sobrevive se para todos os instantes de tempo existe pelo menos uma particula ativa. Caso contrário dizemos que o processo morre.

Observe que $\mathbf{P}_{p}$ [modelo dos sapos sobreviver] é uma função não decrescente de $p$ e definimos

$$
p_{c}(\mathcal{G}):=\inf \left\{p: \mathbf{P}_{p}[\text { modelo dos sapos sobreviver no grafo } \mathcal{G}]>0\right\}
$$

Observe que $p_{c}(\mathcal{G})$ é uma quantidade tal que

$$
\mathbf{P}_{p}[\text { modelo dos sapos sobreviver }]= \begin{cases}=0 & \text { se } p<p_{c} \\ >0 & \text { se } p>p_{c}\end{cases}
$$

$p_{c}(\mathrm{G})$ é chamada probabilidade crítica e dizemos que o modelo apresenta transição de fase caso

$$
0<p_{c}<1 .
$$

Nesta seção apresentamos e provamos resultados sobre transição de fase relacionados ao parâmetro de sobrevivência $p$ na árvore $\mathbf{T}_{d}$. Estamos particularmente interessados no caso em que $\eta(x) \equiv 1$, isto é, no caso em que o número de partículas em cada sítio é igual a 1.

\section{Teorema 4.1}

$$
\frac{k}{2 k-1} \leq p_{c}\left(T_{k}\right) \leq \frac{k}{2 k-4},
$$

para $k \geq 2$, onde $k=d+1$.

Demostração A idéia da prova é devida a Alves [3]. Para o lado esquerdo considere o processo de ramificação em que cada indivíduo gera zero filhos 
com probabilidade $1-p$, um filho com probabilidade $p / k$ e dois filhos com probabilidade $p(k-1) / k$. Observando que a cada instante cada partícula ativa tem pelo menos um sítio vizinho que já foi visitado, podemos acoplar o modelo dos sapos ao processo de ramificação assim definido de forma que o modelo dos sapos é dominado pelo processo de ramificação no sentido de que o número de partículas na geração $n$ deste é sempre maior ou igual ao número de partículas ativas no instante $n$ daquele. Mas o processo de ramificação se extingue quase certamente para $p$ sufucientemente pequeno, o que implica que o modelo dos sapos acoplado a ele também se extingue. Sabemos que o processo de ramificação tem probabilidade positiva de sobreviver se e somente se a esperança do número de filhos for maior do que 1. A partir de uma conta simples verifica-se que o processo de ramificação se extingue se $p \leq k /(2 k-1)$ e como o modelo dos sapos morre se o processo de ramificação morre, devemos ter

$$
p_{c}(\text { modelo dos sapos }) \geq k /(2 k-1) \text {. }
$$

Para o lado direito, a idéia é construir um processo de ramificação em $\mathbf{T}_{k}$ acoplado ao modelo dos sapos, de maneira que o número de indivíduos (partículas) na geração $n$ desse processo seja dominado pelo número de partículas ativas do modelo dos sapos no instante $n$.

No primeiro passo do modelo dos sapos em $\mathbf{T}_{k}$ a partícula da origem (única ativada), sobrevivendo, salta para algum de seus $k=d+1$ vizinhos, que contêm cada um uma partícula desativada. Cada vez que uma partícula 
salta para um sítio ainda não visitado passamos a ter duas partículas ativas nesse sítio. Para distinguí-las denominamo-las primeira e segunda partícula. Diremos que uma partícula vai para frente se ela se afasta da origem, e que ela volta se ela se aproxima da origem (note que o primeiro movimento da partícula da origem, se ela não morrer, é necessariamente para frente). Assim, considere o seguinte processo de ramificação.

\begin{tabular}{c|l|l}
\hline$N^{o}$ Filhos & Quando & Probabilidade \\
\hline $1^{a}$ partícula & & \\
0 & Morre ou salta para trás & $(1-p)+p /(d+1)$ \\
2 & Sobrevive e salta para frente & $\mathrm{pd} /(\mathrm{d}+1)$ \\
\hline $2^{a}$ partícula & & \\
0 & Morre, salta para trás ou salta para frente & \\
2 & para o sítio ocupado pela $1^{a}$ partícula & $\geq(1-p)+p /(d+1)$ \\
& Salta para frente para um sítio não ocupado & $\leq 1-(1-p)+p /(d+1)$ \\
\hline
\end{tabular}

Observe que neste processo, "matamos" todas as partículas do modelo dos sapos que não estão se distanciando da origem e que o número de indivíduos (partículas) na geração $n$ desse processo de ramificação é menor ou igual ao número de partículas ativas do modelo dos sapos que estão a uma distância $n$ da origem. 
As probabilidades associadas ao número de filhos da segunda partícula dependem do que sucede à primeira. Calculamos agora uma aproximação para a probabilidade da segunda partícula gerar dois filhos.

Sejam os eventos:

$A:=\left\{2^{a}\right.$ partícula sobrevive e vai para frente para um sítio não ocupado pela $1^{a}$ partícula $\}$

$\mathrm{e}$

$B:=\left\{1^{a}\right.$ partícula sobrevive e vai para fente $\}$

Então, como $\mathbf{P}(A \mid B) \leq \mathbf{P}\left(A \mid B^{c}\right)$, temos

$$
\mathbf{P}(A)=\mathbf{P}(A \mid B) \mathbf{P}(B)+\mathbf{P}\left(A \mid B^{c}\right) \mathbf{P}\left(B^{c}\right) \geq \mathbf{P}(A \mid B)
$$

Logo, a probabilidade da segunda partícula gerar dois filhos é maior ou igual a $\mathbf{P}(A \mid B)=p(d-1) /(d+1)$.

Temos aqui um processo de ramificação não homogêneo do ponto de vista do número de filhos da primeira e da segunda partícula, já que a probabilidade da segunda ter dois filhos é menor do que a probabilidade da primeira ter dois filhos. Consideremos então um novo processo de ramificação, homogêneo, em que a distribuição do número de filhos das duas partículas é dada por

\begin{tabular}{c|l}
\hline$N^{o}$ Filhos & Probabilidade \\
\hline 0 & $1-p(d-1) /(d+1)$ \\
2 & $p(d-1) /(d+1)$ \\
\hline
\end{tabular}


Esse processo é dominado pelo anterior que por sua vez é dominado pelo modelo dos sapos. É fácil ver que o processo assim definido morre se $p \leq$ $(d+1) / 2(d-1)$, e logo, por comparação, devemos ter

$p_{c}\left(\right.$ modelo dos sapos em $\left.\mathbf{T}_{k}\right) \leq(d+1) / 2(d-1)=k / 2(k-2)$.

Tanto a função do limite inferior como a do limite superior para $p_{c}$ dados pelo teorema são decrescentes em $k$. A partir dos valores dessas funções avaliados para diversos $k$ 's podemos encontrar uma sequência $k_{1}, k_{2}, \cdots$ com $k_{1}<k_{2}<\cdots$ tal que $p_{c}\left(\mathbf{T}_{k_{1}}\right)>p_{c}\left(\mathbf{T}_{k_{2}}\right)>\cdots$.

\subsection{Não Monotonicidade}

Em percolação dizemos que cada elo do grafo está aberto ou fechado com probabilidade $p$ e $1-p$ respectivamente. Dizemos que ocorre o fenômeno de percolação se o vértice denominado origem do grafo pertence a um aglomerado infinito de vértices conectados por elos abertos, o que ocorre se e somente se há uma sequência infinita $x_{0}, x_{1}, x_{2}, \ldots$ de vértices distintos tal que $x_{0}=0, x_{i}$ é vizinho de $x_{i+1}$ e os elos $\left\langle x_{i}, x_{i+1}\right\rangle$ estão abertos para todo i. A questão da sobrevivência no modelo dos sapos pode ser comparada ao fenômeno de percolação da seguinte maneira. Seja $R_{x}$ o conjunto "virtual" de sítios visitados pela partícula originalmente situada em $x . R_{x}$ torna-se "real" caso o sítio $x$ seja visitado por alguma partícula ativa. Então o modelo dos sapos sobrevive se e somente se existir uma sequência infinita de 
sítios distintos $\mathbf{0}=x_{1}, x_{2}, x_{3}, \ldots$ tal que, para todo $i, x_{i+1} \in R_{x_{i}}$. Desta maneira temos um modelo de percolação de conjuntos aleatórios (ver [10]). Desenhando elos orientados desde o vértice $x$ até os elementos de $R_{x}$, temos um modelo de percolação orientada (Durrett [5]).

Assim como no modelo dos sapos, é fundamental na teoria de percolação a existência de um parâmetro crítico $p_{c}=p_{c}(\mathcal{G})$ tal que a probabilidade de haver percolação é zero se $p<p_{c}$ e é maior que zero se $p>p_{c}$ (para mais detalhes sobre a teoria ver Grimmett [7]). Menshikov [11] provou para o modelo de percolação que, sob certas condições $p_{c}\left(\mathcal{G}_{1}\right)<p_{c}\left(\mathcal{G}_{2}\right)$ se $\mathcal{G}_{2}$ é um subgrafo estrito de $\mathcal{G}_{1}$; ele encontrou limites inferiores não triviais para a diferença $p_{c}\left(\mathcal{G}_{2}\right)-p_{c}\left(\mathcal{G}_{1}\right)$.

Mostramos agora que no modelo dos sapos, ao contrário do que ocorre em percolação, é possível apresentar dois grafos $\mathcal{G}_{1}$ e $\mathcal{G}_{2}$ tais que $\mathcal{G}_{1} \subset \mathcal{G}_{2}$ e para os quais $p_{c}\left(\mathcal{G}_{1}\right)<p_{c}\left(\mathcal{G}_{2}\right)$. Para isso, considere o sistema de partículas no grafo $\mathcal{G}_{1}=\mathbb{Z}^{2}$. Agora considere o grafo $\mathcal{G}_{2}^{n}$ constituído por $\mathbb{Z}^{2}$ e por subgrafos completos com $n$ sítios ligados a cada sítio de $\mathbb{Z}^{2}$, de forma que cada sítio de $\mathbb{Z}^{2}$ compõe um subgrafo completo com $n+1$ sítios, conforme ilustrado na Figura 3. Então $\mathcal{G}_{1} \subset \mathcal{G}_{2}^{n}$. Demonstramos que $\lim _{n \rightarrow \infty} p_{c}\left(\mathcal{G}_{2}^{n}\right)=1$, o que, aliado ao fato (Teorema 1.4 em Alves et al. [3]) de que $p_{c}\left(\mathbb{Z}^{2}\right)<1$, implica que existe $n_{0}$ tal que $p_{c}\left(\mathcal{G}_{2}^{n_{0}}\right)>p_{c}\left(\mathcal{G}_{1}\right)$.

Para tanto construímos um processo de ramificação acoplado ao sistema de partículas em $\mathcal{G}_{2}^{n}$ de maneira que o número de partículas na geração $n$ do processo de ramificação é sempre maior ou igual ao número de partículas no instante $n$ do sistema em $\mathcal{G}_{2}^{n}$. 


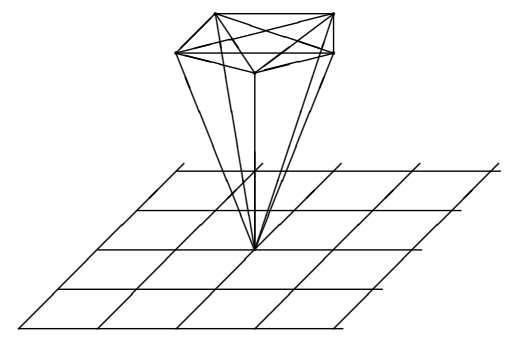

Figura 3: Grafo completo

No sistema em $\mathcal{G}_{2}^{n}$, sempre que uma partícula no nível de $\mathbb{Z}^{2}$ é ativada ela tem possibilidade de saltar para um de seus quatro vizinhos no nível de $\mathbb{Z}^{2}$ e para os $n$ sítios do subgrafo completo conexo. Chamamos de partícula 1 de cada subgrafo aquela originalmente situada no nível de $\mathbb{Z}^{2}$ e as partículas 2 a $n+1$ aquelas originalmente situadas no nível do subgrafo completo. Vamos definir

$X_{i}= \begin{cases}1, & \text { se a } i \text {-ésima partícula do subgrafo completo eventualmente sai do subgrafo } \\ & \text { completo para um dos quatro sítios no nível de } \mathbb{Z}^{2} \text { vizinhos daquele em que } \\ & \text { originalmente se encontra a partícula } 1 \\ 0, & \text { caso contrário }\end{cases}$ e

$$
\begin{aligned}
X=\sum_{i=1}^{n+1} X_{i}= & \text { número de partículas que sai subgrafo para um dos quatro sítios } \\
& \text { no nível de } \mathbb{Z}^{2} \text { vizinhos daquele em que originalmente se encontra } \\
& \text { a partícula } 1
\end{aligned}
$$


No processo original em $\mathcal{G}_{2}^{n}$, quando um partícula é ativada no nível de $\mathbb{Z}^{2}$ por uma outra partícula, essas duas partículas podem, de maneira independente, permanecer no subgrafo completo ativando novas partículas, ou simplesmente sair deste subgrafo. Suponha agora um novo processo em $\mathcal{G}_{2}^{n}$ em que, toda vez que um partícula no nível de $\mathbb{Z}^{2}$ é ativada, são automaticamente ativadas todas as partículas do subgrafo completo conexo ao sítio em que ela se encontra. Nesse instante temos ativadas todas as partículas originalemte situadas nesse subgrafo, inclusive aquela situada no nível de $\mathbb{Z}^{2}$, além da partícula que veio de "fora". Considere então o processo de ramificação cujo número de filhos é dado pelo número de partículas que eventualmente saem do subgrafo completo para um dos seus vizinhos no nível de $\mathbb{Z}^{2}$ neste novo processo. Claramente o processo assim definido domina o processo original em $\mathcal{G}_{2}^{n}$. Agora, no novo processo temos

$$
\mathbf{E}[X]=\mathbf{E}\left[\sum_{i=1}^{n+1} X_{i}\right]=\sum_{i=1}^{n+1} \mathbf{P}\left[X_{i}=1\right] .
$$

Seja $A_{k}$ o evento a partícula sai do subgrafo completo para um de seus vizinhos no nível de $\mathbb{Z}^{2}$ pela primeira vez no $k$-ésimo passo\}, então,

$$
\mathbf{P}\left[X_{i}=1\right]=\sum_{k=1}^{\infty} \mathbf{P}\left[A_{k}\right] .
$$

Para as partículas do subgrafo não situadas no nível de $\mathbb{Z}^{2}$, temos 


$$
\begin{aligned}
\mathbf{P}\left[A_{1}\right] & =0 \\
\mathbf{P}\left[A_{2}\right] & =p \frac{1}{n} p \frac{4}{n+4},
\end{aligned}
$$

e para $k \geq 3$, condicionando no $1^{o}$ passo, temos

$$
\begin{aligned}
\mathbf{P}\left[A_{k}\right]= & \mathbf{P}\left[A_{k} \mid \text { permaneceu no subgrafo }\right] \mathbf{P}[\text { permanecer no subgrafo }] \\
& +\mathbf{P}\left[A_{k} \mid \text { desceu para o nível de } \mathbb{Z}^{2}\right] \mathbf{P}\left[\text { descer para o nível de } \mathbb{Z}^{2}\right]
\end{aligned}
$$

Para $k \geq 3$, se a partícula desce no $1^{o}$ passo ela só pode sair em $k$ passos se no $2^{o}$ passo ela voltar a subir para o subgrafo. Condicionando no $2^{0}$ passo encontramos

$$
\begin{aligned}
& \mathbf{P}\left[A_{k}\right]=\mathbf{P}\left[A_{k-1}\right] p \frac{n-1}{n}+\mathbf{P}\left[A_{k-2}\right] p \frac{1}{n} p \frac{n}{n+4} . \\
& \sum_{k=1}^{\infty} \mathbf{P}\left[A_{k}\right]=\mathbf{P}\left[A_{1}\right]+\mathbf{P}\left[A_{2}\right]+\sum_{k=3}^{\infty} \mathbf{P}\left[A_{k}\right] \\
\sum_{k=3}^{\infty} \mathbf{P}\left[A_{k}\right]= & \sum_{k=3}^{\infty}\left\{\mathbf{P}\left[A_{k-1}\right] p \frac{n-1}{n}+\mathbf{P}\left[A_{k-2}\right] \frac{p^{2}}{n+4}\right\} \\
= & \mathbf{P}\left[A_{2}\right] p \frac{n-1}{n}+\sum_{k=4}^{\infty} \mathbf{P}\left[A_{k-1}\right] p \frac{n-1}{n}+\mathbf{P}\left[A_{2}\right] \frac{p^{2}}{n+4}+\sum_{k=5}^{\infty} \mathbf{P}\left[A_{k-2}\right] \frac{p^{2}}{n+4} \\
= & \left\{p \frac{n-1}{n}+\frac{p^{2}}{n+4}\right\} \mathbf{P}\left[A_{2}\right]+\left\{p \frac{n-1}{n}+\frac{p^{2}}{n+4}\right\} \sum_{k=3}^{\infty} \mathbf{P}\left[A_{k}\right] .
\end{aligned}
$$


Assim,

$$
\sum_{k=3}^{\infty} \mathbf{P}\left[A_{k}\right]=\frac{\left\{p \frac{n-1}{n}+\frac{p^{2}}{n+4}\right\} \mathbf{P}\left[A_{2}\right]}{1-\left\{p \frac{n-1}{n}+\frac{p^{2}}{n+4}\right\}}
$$

Logo, para $i \neq 1$, temos

$$
\begin{aligned}
\mathbf{P}\left[X_{i}=1\right] & =\mathbf{P}\left[A_{1}\right]+\mathbf{P}\left[A_{2}\right]+\sum_{k=3}^{\infty} \mathbf{P}\left[A_{k}\right] \\
& =\mathbf{P}\left[A_{2}\right]\left\{1+\frac{p \frac{n-1}{n}+\frac{p^{2}}{n+4}}{1-\left\{p \frac{n-1}{n}+\frac{p^{2}}{n+4}\right\}}\right\} \\
& =\mathbf{P}\left[A_{2}\right]\left\{\frac{1}{1-\left\{p \frac{n-1}{n}+\frac{p^{2}}{n+4}\right\}}\right\} \\
& =\frac{4 p^{2}}{n(n+4)}\left\{\frac{1}{1-\left\{p \frac{n-1}{n}+\frac{p^{2}}{n+4}\right\}}\right\}
\end{aligned}
$$

Po outro lado,

$$
\mathbf{P}\left[X_{1}=1\right]=\mathbf{P}\left[\text { sair no } 1^{o} \text { passo }\right]+\mathbf{P}[\text { sair em mais de um passo }]
$$

Da mesma maneira, a partícula 1 só pode sair em mais de um passo se no primeiro passo ela subir para o subgrafo completo. Então,

$$
\mathbf{P}\left[X_{1}=1\right]=p \frac{4}{n+4}+\mathbf{P}\left[X_{2}=1\right] p \frac{n}{n+4}
$$

Como duas partículas estão no nível de $\mathbb{Z}^{2}$ no momento em que o subgrafo é ativado, temos para todo $p \in[0,1)$ 


$$
\begin{aligned}
\mathbf{E}[X] & =2 \mathbf{P}\left[X_{1}=1\right]+\sum_{i=2}^{n+1} \mathbf{P}\left[X_{i}=1\right] \\
& =2\left(\frac{4 p}{n+4}+\mathbf{P}\left[X_{2}=1\right] p \frac{n}{n+4}\right)+\sum_{i=2}^{n+1} \mathbf{P}\left[X_{i}=1\right] \\
& =\frac{8 p}{n+4}+\left(2 p \frac{n}{n+4}+n\right) \frac{4 p^{2}}{n(n+4)}\left\{\frac{1}{1-\left\{p \frac{n-1}{n}+\frac{p^{2}}{n+4}\right\}}\right\} \\
& =\frac{8 p}{n+4}+\frac{4 p^{2}(2 p+n+4)}{(n+4)^{2}}\left\{\frac{1}{1-\left\{p \frac{n-1}{n}+\frac{p^{2}}{n+4}\right\}}\right\} \rightarrow 0
\end{aligned}
$$

Resulta daí que $p_{c}\left(\mathcal{G}_{2}^{n}\right) \longrightarrow 1$ quando $n \rightarrow \infty$.

Então, se tomamos $p=p_{c}\left(\mathbb{Z}^{2}\right)+\varepsilon$, com $0<\varepsilon<1-p_{c}\left(\mathbb{Z}^{2}\right)$, podemos encontrar $n_{0}=n_{0}(p)$ tal que a esperança do número de fihos no processo de ramificação acoplado ao modelo dos sapos em $\mathcal{G}_{2}^{n_{0}}$ para esse $p$ seja menor ou igual a 1. Logo, por comparação, a probabilidade do modelo dos sapos sobreviver é zero e devemos ter $p_{c}\left(\mathcal{G}_{2}^{n_{0}}\right) \geq p>p_{c}\left(\mathbb{Z}^{2}\right)$. Portanto esses dois grafos satisfazem $\mathbb{Z}^{2} \subset \mathcal{G}_{2}^{n_{0}}$ e $p_{c}\left(\mathcal{G}_{2}^{n_{0}}\right)>p_{c}\left(\mathbb{Z}^{2}\right)$.

Na Seção 4.1 demonstramos haver $k_{1}$ e $k_{2}$ tais que $\mathbf{T}_{k_{1}} \subset \mathbf{T}_{k_{2}}$ e $p_{c}\left(\mathbf{T}_{k_{2}}\right)<$ $p_{c}\left(\mathbf{T}_{k_{1}}\right)$. Assim, podemos concluir que o parâmetro crítico de sobrevivência do modelo dos sapos não é uma função monótona do grafo. 


\section{Referências}

[1] O.S.M. Alves, F.P. Machado, S.Yu. Popov (2001) The shape theorem for the frog model with random initial configuration. Markov Processes and Related Fields.7, no 4, 525-539.

[2] O.S.M Alves, F.P. Machado, S.Yu. Popov(2002) The shape theorem for the frog model. The Annals of Applied Probability, 12, no 2, 534-547.

[3] O.S.M. Alves, F.P. Machado, S.Yu. Popov (2002) Phase transition for the frog model. Electron. J. Probab. $<$ http://www.math.washington.edu/7Eejpecp $>$ 7, paper No. 16.

[4] K.B. AthreyA, P.E. Ney (1972) Branching Proceses. Springer, Berlin.

[5] R. Durrett (1984) Oriented percolation in two dimensions. Ann. Prob. 12. 12, (4), 999-1040.

[6] R. DuRrett (1988) Lecture Notes on Particle Systems and Percolation. Wadsworth.

[7] G. Grimmett (1999) Percolation. (2nd. ed.) Springer, Berlin.

[8] T.M. Liggett(1985) Interacting Particle Sistems. Springer, New York

[9] T.M. LigGetT(1999) Stochastic interacting systems; contact, voter and exclusion processes. Springer, New York. 
[10] R. Meester, R. RaOy(1996) Continuum percolation. Cambridge University Press, Cambridge.

[11] M.V. Menshikov (1987) Numerical bounds and strict inequalities for critical points of graphs and their subgrafs. Theory of probability and its applications (in Russian) 32, 599-602 (544-547 em tradução).

[12] S.Yu. Popov (2001) Frogs in random environment. J. Statist. Phys. 102 (1/2), 191-201.

[13] H. ThORISson(2000) Coupling, stationarity and regeneration. Springer, New York. 